\title{
Investigation of the stresses exerted by an exosuit of a human arm
}

\author{
Morgan Langard ${ }^{1}$, Yannick Aoustin ${ }^{1}$, \\ Vigen Arakelian ${ }^{1,2}$, and Damien Chablat ${ }^{1}$. \\ ${ }^{1}$ Campus Centrale Nantes, 1, rue de la Noë, BP 92101. 44321 Nantes, France, \\ Laboratoire des Sciences du Numérique \\ de Nantes, UMR CNRS 6004, \\ CNRS, École Centrale de Nantes, Université de Nantes, France \\ ${ }^{2}$ Campus Beaulieu Rennes, INSA, 20, Avenue des Buttes de Coësmes Rennes, \\ France,
}

\begin{abstract}
If a wheelchair could be considered as a better solution than an exoskeleton for the mobility of people suffering from neuromuscular diseases, there is a relevance to use a soft wearable exoskeleton (or exosuit) to assist the upper limbs in order to perform daily tasks such as having a drink, a pencil. It is imperative to limit the stresses generated by the exosuit on human. Numerical tests are proposed to investigate the possible technology choices to design the exoskeleton to limit these stresses. These numerical tests are based on the study of the inverse dynamic model of the human arm and its exosuit. A trajectory of the hand is defined and we deduce the cable tension to track this trajectory. Two decoupled planes are considered for the numerical tests, the sagittal plane where a flexion of the forearm with respect to the upper arm, and the frontal plane where abduction and adduction movements are possible. We assume that the human arm cannot provide any effort. The results show that the position of the anchor points at the shoulder and the orientation of the cable for the abduction movement have an influence to limit the stresses. However, these stresses are important on the shoulder.
\end{abstract}

Keywords: Exosuit, Assistive device, Biped, Energy consumption, Cable tension. 


\section{Nomenclature}

- $m_{i}(\mathrm{~kg})(i=1,2)$ : masses of the upper arm and forearm.

- $I_{i}\left(\mathrm{~kg} \cdot \mathrm{m}^{2}\right)(i=1,2)$ : inertia moments of the upper arm and forearm.

- $\ell_{i}(\mathrm{~m})(i=1,2)$ : lengths of the upper arm and forearm.

- $s_{u}(\mathrm{~m})$ : position of the center of mass of the upper arm with respect the shoulder joint.

- $s_{f}(\mathrm{~m})$ : position of the center of mass of the forearm with respect the shoulder joint.

- $s_{i}(\mathrm{~m})(i=1,2,3)$ : distances between the revolute joint and anchors and attachement point, respectively for the arm and forearm.

- $d_{1}(\mathrm{~m})$ is the height of the anchor and attachement points on the arm.

- $d_{2}(\mathrm{~m})$ is the height of the anchor and attachement points on the shoulder.

- $t_{i}(\mathrm{~N})(i=1,2,3)$ : cable tension.

- $g=9.81 \mathrm{~m} . \mathrm{s}^{-2}$ : Gravity constant.

\section{Introduction}

About 40-50,000 people suffer from neuromuscular diseases (NMD) in France. They are frequently responsible for major handicaps, affecting limb motor function, but also speech, swallowing, and breathing, some of them leading to fatal course. To overcome the evolutive disability of these people the use of a wheelchair for the lower limbs is an efficient solution for the comfort and mobility of patients, even if the wheelchair is not adapted to the human environment. However, there are few solutions for the upper limbs to achieve everyday tasks. A robot manipulator with a robotic hand as effector could be used instead of an assistive arm exoskeleton [8]. However, an arm exoskeleton is more appropriate as the patient can use his dexterity with his hand to achieve some everyday tasks.

Exoskeleton robots are integrated mechanical devices (mechanisms) that can be wearable and attached to the limbs, see for example [6], [10], or [1]. The main role of these mechanical devices is to provide a supplementary strength to avoid muscular disorders and fatigue during the performance of strenuous manual work. An exoskeleton robot could be thus an interesting solution to assist the upper limbs, [9], [5], [13], and [12] for myopathic and neuropathic patients. Rigid exoskeletons show high performance for applications requiring large forces and torques. But they are often heavy equipment, poorly adapted for a patient with weak physical capacities and which 
unfortunately can decrease due to the disease. Furthermore, the assistance of upper limbs is complex due to interaction with the external environment and unpredictable dynamics. The movements of the upper limbs are not also confined to one plane, [2]. Since a few years works exist to compliant exoskeletons called exosuits. Exosuits' intrinsic compliance, portability, and low power consumption make them ideal for providing additional support in activities of daily living such a handing grasping, see for example [3], [4], or [13]. Exosuit actuation is usually based on cable transmission to place the actuator away from the end-effector, despite the friction and backlash phenomenons.

Despite the remarkable progress made in the design of exosuits, the problem of stresses on humans remains open. It is crucial when it comes to patients whose bodies are weakened by disease. We were, therefore, simulating the stresses exerted by an exosuit on the shoulder according to the position of the anchor points in the sagittal plane for bending movements of the arm and in the frontal plane for an abduction movement. Numerical tests show us that it is possible to reduce its constraints according to these technological parameters of the exosuit. These numerical tests are based on the study of the inverse dynamic model of the human arm and its exosuit. A trajectory of the hand is defined and we deduce the cable tension to track this trajectory. We assume that the human arm cannot provide any effort with its muscles. However, the key points are still the exerted stresses on the human. This paper is outlined as follows. Section 2 presents the human arm with its exosuit. The geometric, kinematic and dynamic models of the human arm with its exosuit are recalled in section 3 . The numerical results are analyzed in section 4.2. Section 5 offers our conclusion and perspectives.

\section{Presentation of the concept of the human arm with its exosuit}

We will numerically investigate the human arm with its exosuit in the sagittal plane, Fig. 1 a) and in the frontal plane, Fig. 1 b). The three motors are considered to be fixed to the wheelchair. So we assume that the exosuit has no inertial influence on the movement of the arm, i.e we will consider masses and inertia moments for the human arm only. The cables are considered as inelastic. The points $\mathrm{O}_{1}, \mathrm{O}_{2}$ design the joints of the shoulder and the elbow respectively. The point $O_{3}$ represents the hand with the load. The anchor points $B_{1}, B_{2}$, and $B_{3}$ are attached to the shoulder. So they do not rotate with the arm. The angular parameter $\theta_{3}$ is introduced in the frontal plane to investigate the optimal placement of the motor to perform an abduction movement with the less stresses at the shoulder as possible. 


\begin{tabular}{|c|c|c|c|c|}
\hline & \multicolumn{4}{|c|}{ Human arm } \\
\hline & Mass $(\mathrm{kg})$ & Length $(\mathrm{m})$ & $\begin{array}{c}\text { Inertia moment } \\
\left(\mathrm{kg} \cdot \mathrm{m}^{2}\right)\end{array}$ & $\begin{array}{c}\text { center of } \\
\text { mass }(\mathrm{m})\end{array}$ \\
\hline Upper arm & $m_{u}=2.07$ & $l_{u}=0.364$ & $I^{u}=0.019$ & $s_{u}=0.182$ \\
\hline Forearm & $m_{f}=1.7$ & $l_{f}=0.502$ & $I^{f}=0.043$ & $s_{f}=0.251$ \\
\hline & \multicolumn{4}{|c|}{ Exosuit } \\
\hline
\end{tabular}

Table 1. Physical parameters of a human arm, [11], and the exosuit.

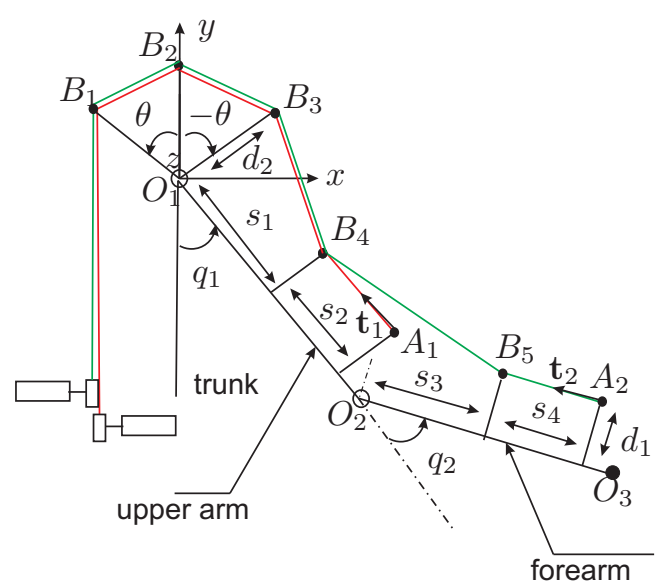

a)



b)

Figure 1. Schemes of the human arm with its exosuit in the sagittal and frontal planes

\section{Models of the human arm with its exosuit}

\subsection{Geometric model}

Let $\left(O_{1}, x, y, z\right)$ be the direct reference frame, see Figs 1 a) and $\left.1 \mathrm{~b}\right)$. Thus the angular variables are counted positive in the counter-clockwise 
direction. We assume that the angular $\theta$ is a constant parameter with the same value for the sagittal and frontal planes. The coordinates of the two joints of the human arm, anchor and attachment points of its exosuit are as follows.

- In the sagittal plane, Fig. 1 a):

$$
\begin{gathered}
\mathbf{O}_{\mathbf{1}} \mathbf{O}_{\mathbf{3}}=\left(\begin{array}{c}
\mathbf{O}_{\mathbf{1}} \mathbf{O}_{\mathbf{3}} \\
\mathbf{O}_{\mathbf{1}} \mathbf{O}_{\mathbf{3}}
\end{array}\right)=\left(\begin{array}{c}
\ell_{1} \sin q_{1}+\ell_{2} \sin \left(q_{1}+q_{2}\right) \\
-\ell_{1} \cos q_{1}-\ell_{2} \cos \left(q_{1}+q_{2}\right)
\end{array}\right), \\
\mathbf{O}_{\mathbf{1}} \mathbf{B}_{\mathbf{1}}=\left(\begin{array}{c}
-d \sin \theta \\
d \cos \theta
\end{array}\right), \\
\mathbf{O}_{\mathbf{1}} \mathbf{B}_{\mathbf{2}}=\left(\begin{array}{c}
0 \\
d
\end{array}\right), \\
\mathbf{O}_{\mathbf{1}} \mathbf{B}_{\mathbf{3}}=\left(\begin{array}{c}
d \sin \theta \\
d \cos \theta
\end{array}\right), \\
\mathbf{O}_{\mathbf{1}} \mathbf{B}_{\mathbf{4}}=\left(\begin{array}{c}
s_{1} \sin q_{1}+d \cos q_{1} \\
-s_{1} \cos q_{1}+d \sin q_{1}
\end{array}\right), \\
\mathbf{O}_{\mathbf{1}} \mathbf{A}_{\mathbf{2}}=\left(\begin{array}{c}
\left(s_{1}+s_{2}\right) \sin q_{1}+d \cos q_{1} \\
-\left(s_{1}+s_{2}\right) \cos q_{1}+d \sin q_{1}
\end{array}\right) \\
\mathbf{O}_{\mathbf{1}} \mathbf{A}_{\mathbf{1}}=\left(\begin{array}{c}
\mathbf{B}_{\mathbf{1}} \sin q_{1}+\left(s_{3}+s_{4}\right) \sin \left(q_{1}+q_{2}\right)+d \cos \left(q_{1}+q_{2}\right) \\
-\ell_{1} \cos q_{1}-\left(s_{3}+s_{4}\right) \cos \left(q_{1}+q_{2}\right)+d \sin \left(q_{1}+q_{2}\right)
\end{array}\right) . \\
\left.\begin{array}{c}
\ell_{1} \sin q_{1}+s_{3} \sin \left(q_{1}+q_{2}\right)+d \cos \left(q_{1}+q_{2}\right) \\
-\ell_{1} \cos q_{1}-s_{3} \cos \left(q_{1}+q_{2}\right)+d \sin \left(q_{1}+q_{2}\right)
\end{array}\right),
\end{gathered}
$$

- In the frontal plane, Fig. 1 a):

$$
\mathbf{O}_{1} \mathbf{O}_{3}=\left(\begin{array}{c}
\left(\ell_{1}+\ell_{2}\right) \sin q_{3} \\
-\left(\ell_{1}+\ell_{2}\right) \cos q_{3}
\end{array}\right) .
$$

\subsection{Inverse geometric model}

- In the sagittal plane (1) we can deduce $q_{1}$ and $q_{2}$ as follows, [7]:

$$
\cos q_{2}=\frac{\mathbf{O}_{\mathbf{1}} \mathbf{O}_{\mathbf{3}}^{2}+\mathbf{O}_{\mathbf{1}} \mathbf{O}_{\mathbf{3}}^{2}-\ell_{1}^{2}-\ell_{2}^{2}}{2 \ell_{1} \ell_{2}},
$$

and then

$$
q_{2}=\operatorname{atan} 2\left( \pm \sqrt{1-\cos ^{2} q_{2}}, \cos q_{2}\right) .
$$




$$
\left\{\begin{array}{c}
\sin q_{1}=\frac{\left(\ell_{1}+\ell_{2} \cos q_{2}\right) \mathbf{O}_{\mathbf{1}} \mathbf{O}_{3 x}+\ell_{2} \sin q_{2} \mathbf{O}_{\mathbf{1}} \mathbf{O}_{\mathbf{3}_{y}}}{\left(\ell_{1}+\ell_{2} \cos q_{2}\right)^{2}+\left(\ell_{2} \sin q_{2}\right)^{2}} \\
\cos q_{1}=\frac{-\left(\ell_{1}+\ell_{2} \cos q_{2}\right) \mathbf{O}_{1} \mathbf{O}_{3 y}+\ell_{2} \sin q_{2} \mathbf{O}_{\mathbf{1}} \mathbf{O}_{\mathbf{3}_{x}}}{\left(\ell_{1}+\ell_{2} \cos q_{2}\right)^{2}+\left(\ell_{2} \sin q_{2}\right)^{2}} \\
q_{1}=\operatorname{atan} 2\left(\sin q_{1}, \cos q_{1}\right) .
\end{array}\right.
$$

- In the frontal plane, by using Fig 1 b) and from (9), $q_{3}$ can be calculated as:

$$
q_{3}=\operatorname{atan} 2\left(\sin q_{3}, \cos q_{3}\right) .
$$

\subsection{Dynamic models}

In the sagittal plane the moments generated by the cables 1 and 2 at the shoulder and elbow respectively are as follows, see [12]:

$$
\begin{aligned}
\mathbf{M}_{\text {shoulder }}^{\text {cable1 }}= & \left(\mathbf{O}_{1} \mathbf{B}_{4} \times\left(\frac{\mathbf{B}_{4} \mathbf{B}_{3}}{\left\|\mathbf{B}_{4} \mathbf{B}_{3}\right\|}+\frac{\mathbf{B}_{4} \mathbf{A}_{1}}{\left\|\mathbf{B}_{4} \mathbf{A}_{1}\right\|}\right)+\ldots\right. \\
& \left.\mathbf{O}_{1} \mathbf{A}_{1} \times\left(\frac{\mathbf{A}_{1} \mathbf{B}_{4}}{\left\|\mathbf{A}_{1} \mathbf{B}_{4}\right\|}\right)\right) t_{1}, \\
\mathbf{M}_{\text {elbow }}^{\text {cable1 }}=\mathbf{0}, & \left(\mathbf{O}_{1} \mathbf{B}_{4} \times\left(\frac{\mathbf{B}_{4} \mathbf{B}_{3}}{\left\|\mathbf{B}_{4} \mathbf{B}_{3}\right\|}+\frac{\mathbf{B}_{4} \mathbf{B}_{5}}{\left\|\mathbf{B}_{4} \mathbf{B}_{5}\right\|}\right)+\ldots\right. \\
\mathbf{M}_{\text {shoulder }}^{\text {cable2 }}= & \mathbf{O}_{1} \mathbf{B}_{5} \times\left(\frac{\mathbf{B}_{5} \mathbf{B}_{4}}{\left\|\mathbf{B}_{5} \mathbf{B}_{4}\right\|}+\frac{\mathbf{B}_{5} \mathbf{A}_{2}}{\left\|\mathbf{B}_{5} \mathbf{A}_{2}\right\|}\right)+\ldots \\
& \left.\mathbf{O}_{1} \mathbf{A}_{2} \times\left(\frac{\mathbf{A}_{2} \mathbf{B}_{5}}{\left\|\mathbf{A}_{2} \mathbf{B}_{5}\right\|}\right)\right) t_{2}, \\
\mathbf{M}_{\text {elbow }}^{\text {cable2 }}= & \left(\mathbf{O}_{2} \mathbf{B}_{5} \times\left(\frac{\mathbf{B}_{5} \mathbf{B}_{4}}{\left\|\mathbf{B}_{5} \mathbf{A}_{4}\right\|}+\frac{\mathbf{B}_{5} \mathbf{A}_{2}}{\left\|\mathbf{B}_{5} \mathbf{A}_{2}\right\|}\right)+\ldots\right. \\
& \left.\mathbf{O}_{2} \mathbf{A}_{2} \times\left(\frac{\mathbf{A}_{2} \mathbf{B}_{5}}{\left\|\mathbf{A}_{2} \mathbf{B}_{5}\right\|}\right)\right) t_{2} .
\end{aligned}
$$

We can deduce the equivalent joint torques, which are developed by $t_{1}$ and $t_{2}$ at the shoulder and elbow:

$$
\begin{aligned}
\left(\begin{array}{c}
\Gamma_{1} \\
\Gamma_{2}
\end{array}\right) & =\left(\begin{array}{c}
\mathbf{M}_{\text {shoulder }}^{\text {cable1 }}+\mathbf{M}_{\text {shoulder }}^{\text {cable2 }} \\
\mathbf{M}_{\text {elbow }}^{\text {cable2 }}
\end{array}\right) \\
& =\quad \mathbf{J}_{c 1}^{\top}\left(\begin{array}{c}
t_{1} \\
t_{2}
\end{array}\right),
\end{aligned}
$$


where the Jacobian matrix $\mathbf{J}_{c 1}$ is a square matrix $(2 \times 2)$. In the sagittal plane the dynamic model of the arm and its exosuit can be written such as:

$$
\mathbf{D} \ddot{\mathbf{q}}+\mathbf{h}(\mathbf{q}, \dot{\mathbf{q}}) \dot{\mathbf{q}}+\mathbf{G}=\mathbf{J}_{c 1}^{\top}\left(\begin{array}{c}
t_{1} \\
t_{2}
\end{array}\right)
$$

Here $\mathbf{q}=\left(q_{1}, q_{2}\right)^{\top}$ is the generalized vector, $\mathbf{D}(2 \times 2)$ is the inertia matrix, $\mathbf{h}(2 \times 2)$ represents the centrifugal and Coriolis effects and $\mathbf{G}(2 \times 1)$ defines the gravity force.

In the frontal plane, Fig. 1 b), the moment generated by the cable 3 at the shoulder is:

$$
\begin{aligned}
\mathbf{M}_{\text {shoulder }}^{\text {cable3 }} & =\mathbf{O}_{\mathbf{1}} \mathbf{A}_{\mathbf{3}} \times\left(\frac{\mathbf{A}_{\mathbf{3}} \mathbf{B}_{\mathbf{3}}^{\prime}}{\left\|\mathbf{A}_{\mathbf{3}} \mathbf{B}_{\mathbf{3}}^{\prime}\right\|}\right) t_{3} \\
& =\mathbf{J}_{c 2}^{\top} t_{3} .
\end{aligned}
$$

Here the Jacobian matrix $\mathbf{J}_{c 2}(1 \times 1)$ is a scalar matrix. Similarly to the sagittal plane the dynamic model of the arm and its exosuit in the frontal plane can be written with a scalar equation such as:

$$
\mathbf{D} \ddot{q}_{3}+\mathbf{G}=\mathbf{J}_{c 2}^{\top} t_{3} .
$$

Here $\mathrm{D}(1 \times 1)$ is the inertia moment with respect to the center of mass of the arm with its exosuit and the load and $\mathrm{G}(1 \times 1)$ defines the gravity force.

\subsection{Kinematic models}

These models are useful to compute $\dot{\mathbf{q}}$ and $\ddot{\mathbf{q}}$ in the inverse dynamic model to determine the cable tensions.

- In the sagittal plane, considering the time derivative of (1) the first order kinematic model and the second order kinematic model can be written such as:

$$
\begin{aligned}
& \frac{\mathrm{d} \mathbf{O}_{1} \mathbf{O}_{3}}{\mathrm{~d} t}=\left(\begin{array}{l}
\mathbf{O}_{1} \dot{\mathbf{O}_{3}} \\
\mathbf{O}_{1} \mathbf{O}_{3 y}
\end{array}\right) \\
& =\quad \mathbf{J} \dot{\mathbf{q}} \\
& \frac{\mathrm{d}^{2} \mathbf{O}_{1} \mathbf{O}_{3}}{\mathrm{~d} t^{2}}=\mathbf{J} \ddot{\mathbf{q}}+\dot{\mathbf{J}} \dot{\mathbf{q}}
\end{aligned}
$$

- In the frontal plane the principle is similar, considering that the trajectory point $O_{3}$ describes a circle. With the first time derivative and second time derivative of this equation we can calculate $\dot{q}_{3}$ and $\ddot{q}_{3}$. 


\section{Numerical results}

\subsection{Numerical results in the sagittal plane}

A reference trajectory for $\mathrm{O}_{3}$ is defined in the Cartesian space by prescribing a five order polynomial function for $\mathbf{O}_{\mathbf{1}} \mathbf{O}_{\mathbf{3} x}$ and for $\mathbf{O}_{\mathbf{1}} \mathbf{O}_{\mathbf{3}}$ respectively. We are able to define the initial and final positions, velocities and accelerations for the human arm with its exosuit along $x$ - axis and $y$ - axis. By using the inverse geometric models (11), (13) and the kinematic models (23), (24) we calculate the components $q_{i}, \dot{q}_{i}$, and $\ddot{q}_{i}$ with $i=1,2$, the left part of (20) and then finally the cable tensions $t_{1}$ and $t_{2}$. By varying the parameter $s_{1}$ and $s_{3}$, we investigate the norm of the net force $\mathbf{F}$, Figs 2 and the net moment acting on the human shoulder Figs 3, without load and with a load of $0.5 \mathrm{~kg}$ in the hand $\left(\mathrm{O}_{3}\right)$. The net force and the net moment are due to the cable tension $t_{1}$ and $t_{2}$ in the anchor points $B_{1}, B_{2}, B_{3}$. They represent the stresses that the shoulder has to compensate. We observe in Fig. 2 b) the influence of the load because the norm of the net force is more important than in Fig. 2 a), that is logical. If the parameter $s_{1}$ has a great influence, the net force acting on the shoulder does not seem to depend on $s_{3}$. However the minimal modulus of the net force is almost $150 \mathrm{~N}$ without load and $250 \mathrm{~N}$ with the load. This represents a great stresses for a children, who suffers of a neuromuscular disease. Fig. 3 shows that the net moment does not also depend on the parameter $s_{3}$. Its norm is close to zero. This means that the exosuit stays firmly on the shoulder without rotation.

\subsection{Numerical results in the frontal planel}

The trajectory of the tip point $O_{3}$ being a circle in the Cartesian space a polynomial function of five order is defined for $q_{3}$. Then it is possible to calculate $\dot{q}_{3}$ and $\ddot{q}_{3}$, the left part of (3.3) and then finally the cable tension $t_{3}$. The parameter $s_{3}$ is equal to $0.9\left(\ell_{1}+\ell_{2}\right)$. Figures (4) a) and b) show the norms of the net force and the net moment for an abduction movement due to the cable tension $t_{3}$ at the anchor points $B_{1}^{\prime}, B_{2}^{\prime}$, and $B_{1}^{\prime} 3$ at the shoulder. There is a positive influence of $\theta_{3}$ because the norm of the net force is minimal and the corresponding norm of the net moment is near 1 N.m. But as in the sagittal plane, the minimal norm of the net force is still important for a children. 


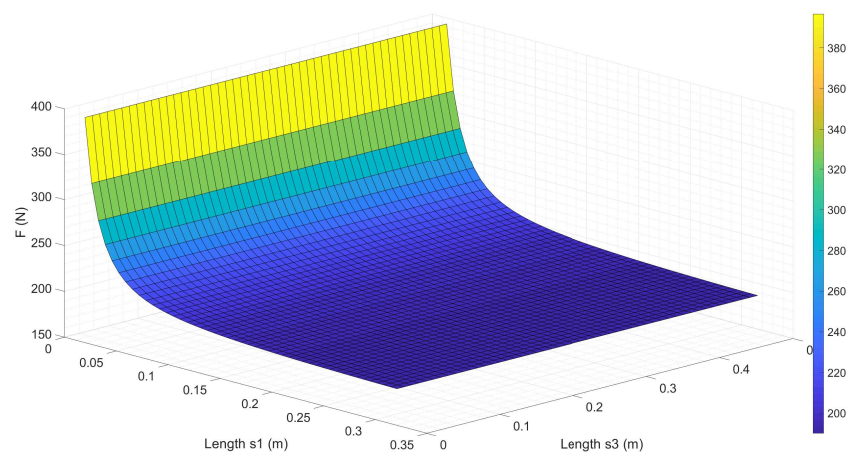

a)

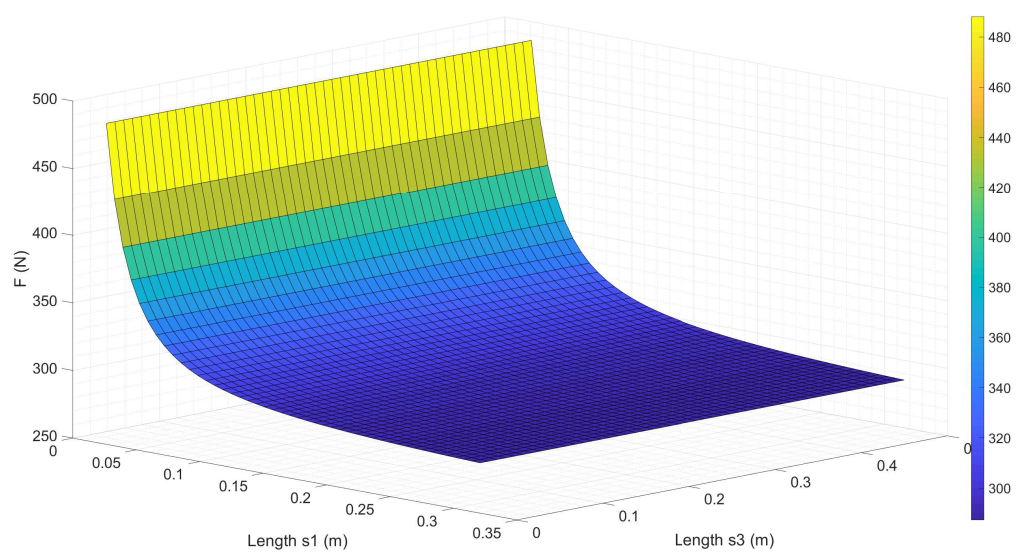

b)

Figure 2. Norm of the net force $F$ as function of the parameters $s_{1}$ and $s_{3}$, without load Fig. 2 a) and with a load Fig. 2 b) 


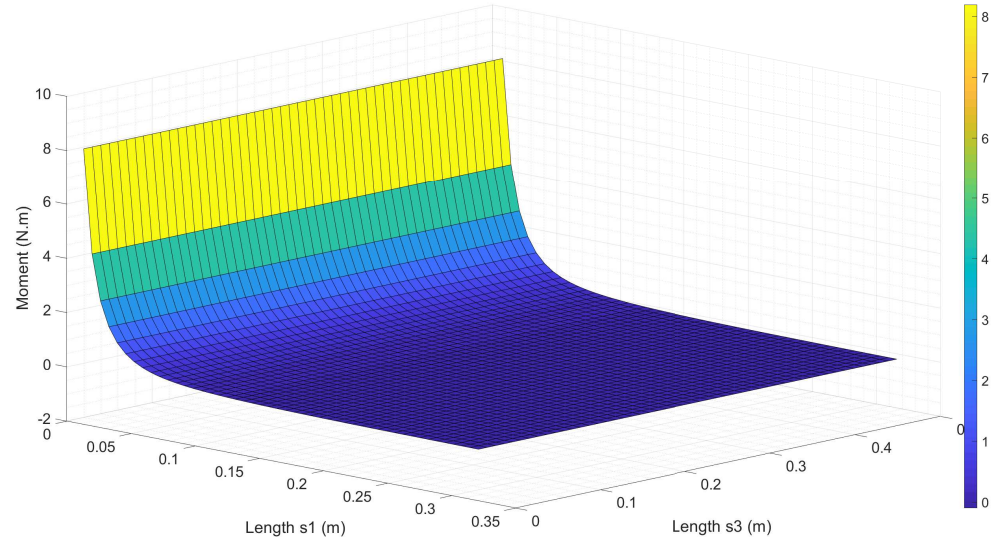

a)

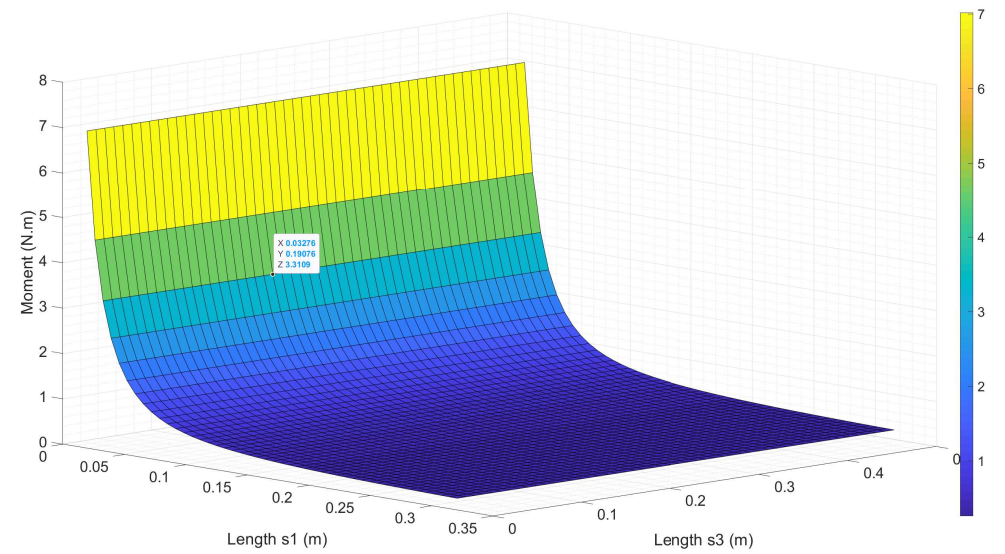

b)

Figure 3. Norm of the net Moment as function of the parameters $s_{1}$ and $s_{3}$ 


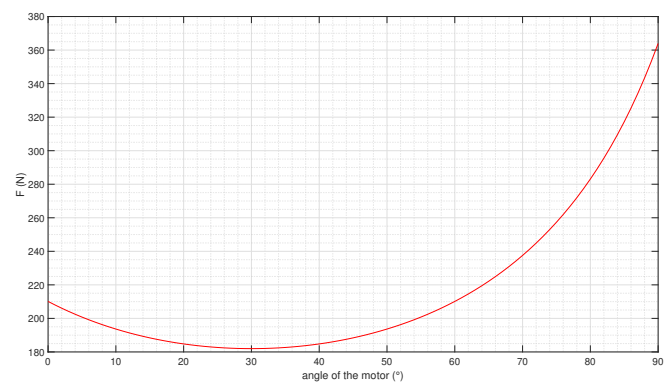

a)

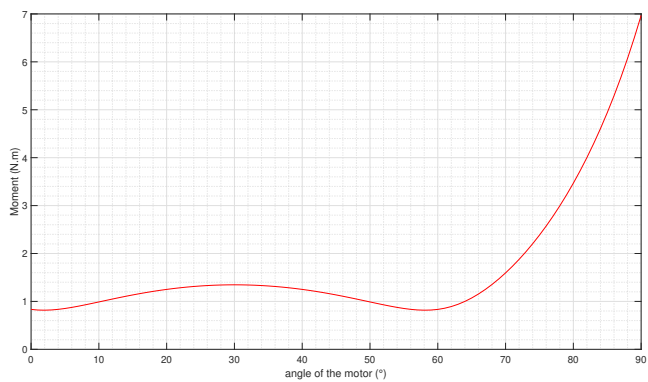

b)

Figure 4. Norm of the net force and net Moment as function of $\theta_{3}$ for an abduction movement

\section{Conclusion and perspectives}

The distribution of the anchor and attachement points can limit the stresses from the exosuit to the human shoulder. The position of the actuators on the wheelchair is also important to improve the performance of the exoskeleton, especially for the abduction and adduction movements. Despite these results the stresses due to the exosuit on the shoulder is still important. However we assume that the human arm cannot provide any efforts with its muscles. The exosuit is probably more adapted to assist partially human to carry out a task. The perspectives are to investigate the association of springs and cables for the exosuit and to consider different distributions of the anchor and attachement points. We plane also to extend this work with a 3D dynamic model with a new shape of the exosuit at the shoulder. 


\section{Bibliography}

[1] Y. Aoustin and A. M. Formalskii. Strategy to Lock the Knee of Exoskeleton Stance Leg: Study in the Framework of Ballistic Walking Model. New Trends in Medical and Service Robots: Human Walking, Eds. P. Wenger, C. Chevallereau, Doina Pisla, Hannes Bleuler, and A. Rodic. 275p, 2016.

[2] B. Bennett, J. M. Hollerbach, Y. Xu, and I. Hunter. Time-varying stiffness of human elbow joint during cyclic voluntary movement. Exp. Brain Res., 88(2):433-1442, 1992.

[3] L. Cappelo, D. Khanh Binh, S. C. Yen, and L. Masiai. Design and preliminary characterization of a soft wearable exoskeleton for upper limb. In Proc. 6th IEEE RAS/EMBS Int. Conf. on Biomedical Robotics and Biomechatronics (BioRob), pages 623-629, June 26-29, UTown, Singapore, 2016.

[4] B. Khanh Dinh, M. Xiloyannis, C. Wilson Antuvan, L. Cappello, and L. Masia. Hierarchical cascade controller for assistance modulation in a soft wearable arm exoskeleton. IEEE robotics and automation letters, 2(3):1786-1793, 2017.

[5] M. Gunn, T. M. Shank, M. Epps, J. Hossain, and T. Rahman. User evaluation of a dynamic orthosis for people with neuromuscular disorders. IEEE Trans. On Neural systems and rehabilitation engineering, 24(12):1277-1283, 2016.

[6] H. Herr. Exoskeletons and orthoses: classification, design, design challenges and future directions. J. of NeuroEngineering and Rehabilitation, 6:21:1-9, doi:10.1186/1743-0003-6-21, 2009.

[7] W. Khalil and E. Dombre. Modeling, identification and control of robots. Butterworth Heinemann, 2002.

[8] I. Papadakis Ktistakis and N. G. Bourbakis. A survey on robotic wheelchairs mounted with robotic arms. In Proc. National aerospace and electronics conference (NAECON), pages 258-262, 2015.

[9] J. C. Perry, J. Rosen, and S. Burns. Upper-limb powered exoskeleton design. IEEE/ASM Trans. On Mechatronics, 12(4):408-417, 2007.

[10] B. S. Rupala, A. Singla, and G. S. Virk. Lower limb exoskeletons: A brief review. In Proc. Int. Conf. on Mechanical Engineering \& Technology COMET, pages 18-24, Varanasi, Utter Pradesh, 2016.

[11] D. A. Winter. Biomechanics and motor control of human movement. Jonhn Wiley and Sons, 2009.

[12] Y. Zhang. Design and optimization of a new robotic suit for load carriage via coupling cables and a rigid support. PhD thesis, INSA RENNES France, 2019. 
[13] Y. Zhang and V. Arakelian. Design of a passive robotic exosuit for carrying heavy loads. In Proc. IEEE Int. Conf. on Humanoid robots (Humanoids), pages 860-865, Beijing, China, 2018. 